\title{
Prospects of indirect searches for dark matter annihilations in the earth with ICAL@INO
}

\author{
Deepak Tiwari, ${ }^{a}$ Sandhya Choubey ${ }^{a, b}$ and Anushree Ghosh ${ }^{c}$ \\ ${ }^{a}$ Harish-Chandra Research Institute (HBNI), \\ Chhatnag Road, Jhunsi, Allahabad 211 019, India \\ ${ }^{b}$ Department of Physics, School of Engineering Sciences, KTH Royal Institute of Technology, \\ AlbaNova University Centre, 10691 Stockholm, Sweden \\ ${ }^{c}$ Universidad Tecnica Federico Santa Maria, Departamento de Fisica, \\ Casilla 110-V, Avda. Espana 1680, Valparaiso, Chile \\ E-mail: deepaktiwari@hri.res.in, sandhya@hri.res.in, \\ anushree.ghosh@usm.cl
}

ABSTRACT: We study the prospects of detecting muon events at the upcoming Iron CALorimeter (ICAL) detector to be built at the proposed India-based Neutrino Observatory (INO) facility due to neutrinos arising out of annihilation of Weakly Interactive Massive Particles (WIMP) in the centre of the earth. The atmospheric neutrinos coming from the direction of earth core presents an irreducible background. We consider 50kt $\times$ 10 years of ICAL running and WIMP masses between $10-100 \mathrm{GeV}$ and present $90 \%$ C.L. exclusion sensitivity limits on $\sigma_{\mathrm{SI}}$ which is the WIMP-nucleon Spin Independent (SI) interaction cross-section. The expected sensitivity limits calculated for ICAL for the WIMP annihilation in the earth are more stringent than the limits obtained by any other neutrino detector. For a WIMP mass of $52.14 \mathrm{GeV}$, where the signal fluxes are enhanced due to resonance capture of WIMP in earth due to Fe nuclei, the sensitivity limits, assuming $100 \%$ branching ratio for each channel, are: $\sigma_{\mathrm{SI}}=3.43 \times 10^{-45} \mathrm{~cm}^{2}$ for $\nu_{\mu} \overline{\nu_{\mu}}$ channel, $\sigma_{\mathrm{SI}}=1.02 \times 10^{-44} \mathrm{~cm}^{2}$ for $\tau^{+} \tau^{-}$channel and $\sigma_{\mathrm{SI}}=5.36 \times 10^{-44} \mathrm{~cm}^{2}$ for $b \bar{b}$ channel.

Keywords: Dark matter, Neutrino Detectors and Telescopes (experiments)

ArXiv EPrint: 1806.05058 


\section{Contents}

1 Introduction 1

2 Neutrino flux from WIMP annihilation in the earth 2

3 Event generation at ICAL $\quad 6$

4 Atmospheric neutrino background suppression $\quad 8$

$\begin{array}{lll}5 & \text { The statistical analysis } & 11\end{array}$

6 Results 12

$\begin{array}{lll}7 & \text { Summary } & 15\end{array}$

\section{Introduction}

The Weakly Interacting Massive Particles (WIMP) have been proposed as one of the leading particle dark matter candidates to explain the missing non-luminous matter of the universe [1-3]. The WIMP with masses in the mass range of a few GeVs to tens of $\mathrm{TeV}$ would get gravitationally attracted to the celestial body, scatter off the nucleons in the celestial bodies and lose energy. WIMP whose velocities become less than the escape velocity of the celestial body are then trapped in the gravitational potential well of the celestial body. The trapped WIMP eventually sink to the centre of the celestial body due to gravity where their concentration increases. Subsequent annihilations of WIMP is expected to produce neutrinos in their final states. These neutrinos are expected to come with an energy spectrum in the range $\left[0-m_{\chi}\right]$, where $m_{\chi}$ is the WIMP mass. These neutrinos can be detected in the neutrino detectors, providing an indirect evidence for the existence of WIMP dark matter. Such indirect detection signals for WIMP in the sun [4-6] and earth $[6,7]$ have been looked for in the currently running neutrino detectors such as IceCube [4], Antares [6] and Super-Kamiokande (SK) [5]. Since none of the detectors have recorded any positive signal for WIMP annihilations in the sun and earth, they have given exclusion limits in the WIMP scattering cross-section - WIMP mass space.

The magnetised Iron CALorimeter (ICAL) detector proposed to be built at the Indiabased Neutrino Observatory (INO) should be able to detect the neutrinos from WIMP annihilations if the WIMP indeed have masses in the few $\mathrm{GeV}$ to $100 \mathrm{~s}$ of $\mathrm{GeV}$ range. In our previous work [8] we explored the prospects of indirect detection of WIMP annihilation in the sun at the ICAL detector at INO. In this work we study the prospect of indirect detection of WIMP at ICAL from their annihilations in the centre of the earth. As is well known, the WIMP annihilation cross-section can be related to the WIMP-nucleus scattering 
cross-section. The WIMP scattering on nucleons can proceed both via Spin Independent (SI) as well as Spin Dependent (SD) process, where the SI cross-section depends on the mass of the nucleus involved while the SD cross-section does not. Rather, SD WIMP nucleon scattering is relevant only for nuclei which have a non-zero spin in their ground state and therefore, only the target nuclei with unpaired nucleons are sensitive to SD scattering cross section. Since the sun has a large abundance of hydrogen, SD scattering and capture is significant and hence the neutrino detectors looking for dark matter annihilation in the sun have provided competetive bounds on SD WIMP-proton cross section. The earth, however, has only a negligible fraction of nuclei with spin and hence SD scattering is not relevant for the case of the earth [9]. On the other hand, since heavier target nuclei offer better sensitivity to SI cross-section, the earth can be sensitive to SI cross-sections. Similarly, the WIMP direct detection experiments, which look for the recoil energy of target nuclei due to WIMP scattering on them in dedicated terrestrial detectors, are more sensitivity to SI or SD cross-section, depending on the nature of their target material.

While the direct detection experiments continue to be several order of magnitude more sensitive for SI interactions, indirect searches provide a complementary probe to dark matter and are essential to fully reconstruct the particle nature of dark matter.

The organisation of the paper is as follows. In section 2 we calculate the signal neutrino spectra due to WIMP annihilation in the earth. In section 3 we describe the detector and the event generation procedure. Thereafter, in section 4, we describe the atmospheric background suppression scheme. In section 5 we describe our statistical analysis, present results in section 6 and finally conclude in section 7 .

\section{Neutrino flux from WIMP annihilation in the earth}

The number of WIMP $(N)$ inside the earth as a function of time $t$ is given by the following differential equation $[9]$,

$$
\frac{d N}{d t}=C-C_{\mathrm{A}} N^{2}-E N,
$$

where the terms on the right-hand side correspond to capture of WIMP inside the earth $(C)$, annihilation in the core $\left(C_{\mathrm{A}}\right)$ and evaporation from its surface $(E)$, respectively. In this work we neglect the effect of evaporation from the earth assuming that it is not significant for reasonably heavy WIMP [10-13]. Each annihilation reduces the number of WIMP by two units and hence the rate of depletion of WIMP is twice the annihilation rate in the earth,

$$
\Gamma_{\mathrm{A}}=\frac{1}{2} C_{\mathrm{A}} N^{2}
$$

The quantity $C_{\mathrm{A}}$ is a function of total WIMP annihilation cross-section times the relative velocity of the WIMP $\left(\left\langle\sigma_{\mathrm{A}} v\right\rangle\right)$ and the distribution of WIMP in the earth and can be written as $[9,14]$,

$$
C_{\mathrm{A}}=\left\langle\sigma_{\mathrm{A}} v\right\rangle \frac{V_{2}}{V_{1}^{2}},
$$

where $V_{j}$ is the effective volume of the earth and is given by $V_{j}=2 \times 10^{25}\left(j m_{\chi}^{10}\right)^{3 / 2} \mathrm{~cm}^{3}$, and $m_{\chi}^{10}$ being WIMP mass in the units of $10 \mathrm{GeV}[9,14]$. Solving eq. (2.1) for $N$, we find 
the annihilation rate at any given time as,

$$
\Gamma_{\mathrm{A}}=\frac{1}{2} C \tanh ^{2}(t / \tau),
$$

where $\tau$ is the time required for equilibrium to be established between the capture and annihilation of WIMP in the earth and is related to $C$ and $C_{\mathrm{A}}$ through the expression

$$
\tau=\left(C C_{\mathrm{A}}\right)^{-1 / 2}
$$

In case $t \gg \tau$, equilibrium would have reached and the relation $\Gamma_{A}=C / 2$ would hold. For a $50 \mathrm{GeV}$ WIMP, assuming $\left\langle\sigma_{\mathrm{A}} v\right\rangle=3 \times 10^{-26} \mathrm{~cm}^{3} \mathrm{~s}^{-1}$ in eq. $(2.3)$, and $\sigma_{\mathrm{SI}}=10^{-44} \mathrm{~cm}^{2}$ in the capture rate expression (eq. (2.7)), we get value of $C_{\mathrm{A}}$ and $C$, respectively. Putting these values of $C_{\mathrm{A}}$ and $C$ in from eq. (2.5), we get $\tau \sim 10^{18} \mathrm{~s}$. For WIMP masses less than $50 \mathrm{GeV}$, the value of $\tau$ increases by a few orders of magnitude. For masses above $50 \mathrm{GeV}$ it is seen to increase by an order of magnitude. For the age of the earth, which of the order of $\sim 10^{17} \mathrm{~s}$, we therefore see that equilibrium between the annihilation and capture rate is never achieved and hence $\Gamma_{A} \neq C / 2$. Nevertheless, $t / \tau$ can be related to the capture rate $C$ and annihilation cross-sections $\left\langle\sigma_{\mathrm{A}} v\right\rangle$ via the following relation [9]:

$$
\frac{t_{\oplus}}{\tau}=1.9 \times 10^{4}\left(\frac{C}{\mathrm{~s}^{-1}}\right)^{1 / 2}\left(\frac{\left\langle\sigma_{\mathrm{A}} v\right\rangle}{\mathrm{cm}^{3} \mathrm{~s}^{-1}}\right)^{1 / 2}\left(\frac{m_{\chi}}{10 \mathrm{GeV}}\right)^{3 / 4}
$$

where $t=t_{\oplus} \sim 4.5 \times 10^{9}$ years is the age of the earth. From eq. (2.3), we can see that for a given WIMP mass and for a fixed value of $\left\langle\sigma_{\mathrm{A}} v\right\rangle, C_{\mathrm{A}}$ is constant and using eq. (2.4) a direct proportionality between $\Gamma_{\mathrm{A}}$ and $C$ can be established. Hence, the annihilation of WIMP in earth can be related to the WIMP-nucleon scattering cross section. The scattering of the WIMP could proceed via both Spin Dependent (SD) and Spin Independent (SI) processes. The SI scattering cross-section depends on the number of nucleons present in the nucleus and hence is dominant for heavy nuclei. Since the earth comprises mainly heavy nuclei, the SI WIMP-nucleon scattering is dominant and is given by $[9,15]$ :

$$
C=c\left(\frac{1 \mathrm{GeV}}{m_{\chi}}\right)\left(\frac{\rho_{\text {local }}}{0.3 \mathrm{GeV} / \mathrm{cm}^{3}}\right)\left(\frac{270 \mathrm{~km} / \mathrm{s}}{\bar{v}_{\text {local }}}\right) \sum_{i} F_{i}\left(m_{\chi}\right) \sigma_{\mathrm{SI}}^{i} f_{i} \phi_{i} \frac{S\left(m_{\chi} / m_{N i}\right)}{m_{N i} /(1 \mathrm{GeV})},
$$

where $c$ is a constant with value $4.8 \times 10^{15} \mathrm{~s}^{-1}$ for the case of earth and $m_{\chi}$ is the mass of WIMP. $\rho_{\text {local }}$ and $\bar{v}_{\text {local }}$ are the local DM density and velocity dispersion in the halo respectively. The summation in eq. (2.7) has to be carried out over all the nuclei in the earth where $F_{i}\left(m_{\chi}\right)$ is the form-factor suppression for the capture of a WIMP of mass $m_{\chi}$ with the $i^{\text {th }}$ nucleus. For the $i^{\text {th }}$ nuclear species with mass $m_{N_{i}}$ (in GeV), $f_{i}$ is the mass fraction of the $i^{\text {th }}$ element. The normalised average "potential energy" $\phi_{i}$ depends on the spatial distribution of the $i^{\text {th }}$ element in the earth and is expressed as $\phi_{i}=\left\langle v^{2}\right\rangle_{i} / v_{0}$, where $\left\langle v^{2}\right\rangle_{i}$ being the escape velocity square of WIMP, averaged over the distribution of the element $i$ and $v_{0}$ is the escape velocity at the surface of the earth $[9,15] . \sigma_{\mathrm{SI}}^{i}$ is the cross-section for elastic scattering of the WIMP on $i^{\text {th }}$ nuclear species via SI interaction in the units of $10^{-40} \mathrm{~cm}^{2}$. For the capture of the WIMP on $i^{\text {th }}$ nuclei, $S\left(m_{\chi} / m_{N_{i}}\right)$ gives 


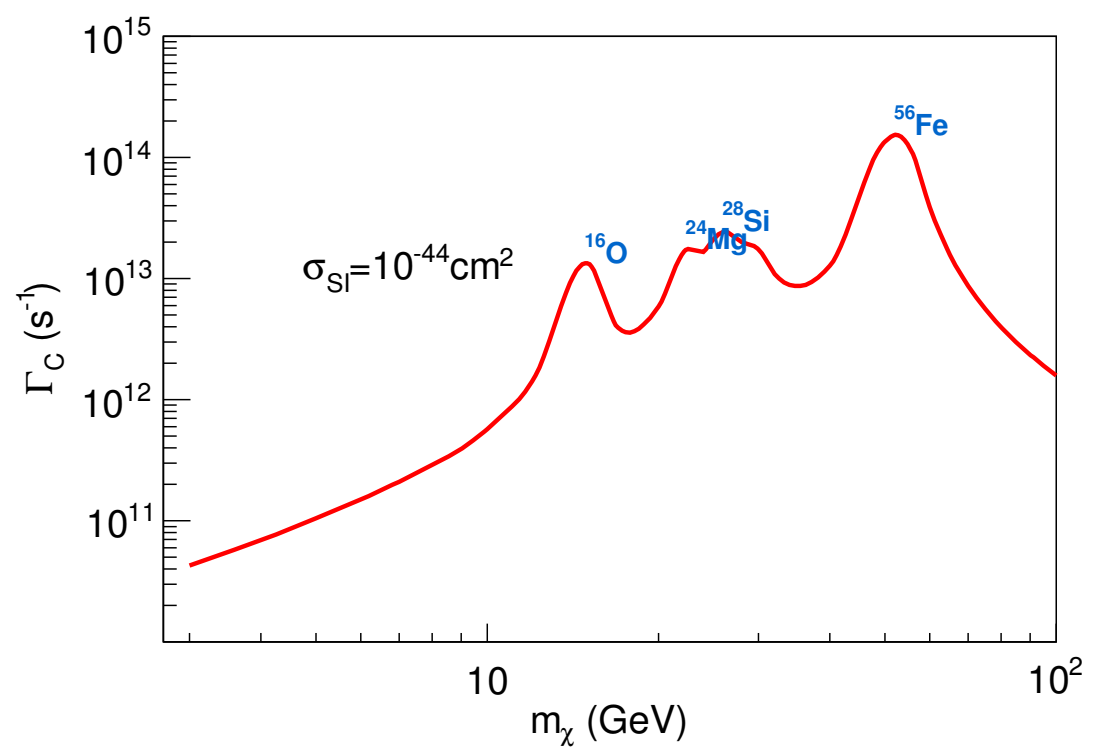

Figure 1. SI capture rate for WIMP in the earth as a function of WIMP mass $m_{\chi}$. The enhanced capture rate due to resonance scattering on various nuclei is also shown.

the corresponding kinematic suppression factor. The cross-section for interaction of WIMP $\sigma_{\mathrm{SI}}^{i}$ with $i^{\text {th }}$ nucleus can be related to WIMP-nucleon interaction cross-section $\sigma_{\mathrm{SI}}$ by the following expression:

$$
\sigma_{\mathrm{SI}}^{i}=\sigma_{\mathrm{SI}} A_{i}^{2}\left(\frac{\mu_{\chi N_{i}}}{\mu_{\chi p}}\right)^{2}
$$

where for the $i^{\text {th }}$ nucleus: $A_{i}$ is the atomic number, $\mu$ is its reduced mass and $m_{N_{i}} \approx$ $A_{i} m_{p}, m_{p}$ being the proton's mass. Here we assume the proton mass to be equal to the neutron mass. The annihilation of WIMP produce standard model particle antiparticle pairs. Subsequently, hadronisation and/or decay of these pair products can give rise to neutrinos. Due to WIMP annihilation in the earth, the differential neutrino flux arising at the detector is given by:

$$
\frac{d N_{\nu}^{\prime}}{d \Omega d t d E_{\nu}}=\frac{\Gamma_{\mathrm{A}}}{4 \pi R^{2}} \sum_{j=1} \mathrm{BR}_{j} \frac{d N_{j}}{d E_{\nu}},
$$

where $\Gamma_{A}$ and $C$ are related as discussed above, $R$ is the distance travelled by the neutrinos between the point of creation in the earth's core and detection at the detector. $d N_{j} / d E_{\nu}$ is the differential neutrino flux for a given WIMP annihilation channel $j$ such as $W^{+} W^{-}$, $b \bar{b}, c \bar{c}, \tau^{+} \tau^{-}$etc. The sum in eq. (2.9), which is over all possible channels $j$, has to be weighted with the branching ratio $\left(B R_{j}\right)$ of the particular channel $j$. Considering a generic WIMP scenario, we take one annihilation channel at a time and assume $100 \%$ branching ratio for each of the channels. For a specific model predicting a different $B R_{j}$, the above fluxes would be simply mixture of different channels scaled linearly.

The annihilation of WIMP into standard model particle-antiparticle pairs in the centre of the earth, followed by the propagation of the produced neutrinos up to the detector is done using the WIMPSIM $[16,17]$ package. WIMPSIM uses Nusigma [18] for simulation of 


\begin{tabular}{|lr|}
\hline Paramter & Best-Fit Value \\
\hline$\theta_{12}$ & $34^{\circ}$ \\
$\theta_{13}$ & $9.2^{\circ}$ \\
$\theta_{23}$ & $45^{\circ}$ \\
$\delta$ & 0 \\
$\Delta m_{21}^{2}$ & $7.5 \times 10^{-5} \mathrm{eV}^{2}$ \\
$\Delta m_{31}^{2}$ & $2.4 \times 10^{-3} \mathrm{eV}^{2}$ \\
\hline
\end{tabular}

Table 1. Oscillation parameters used in the Simulation.

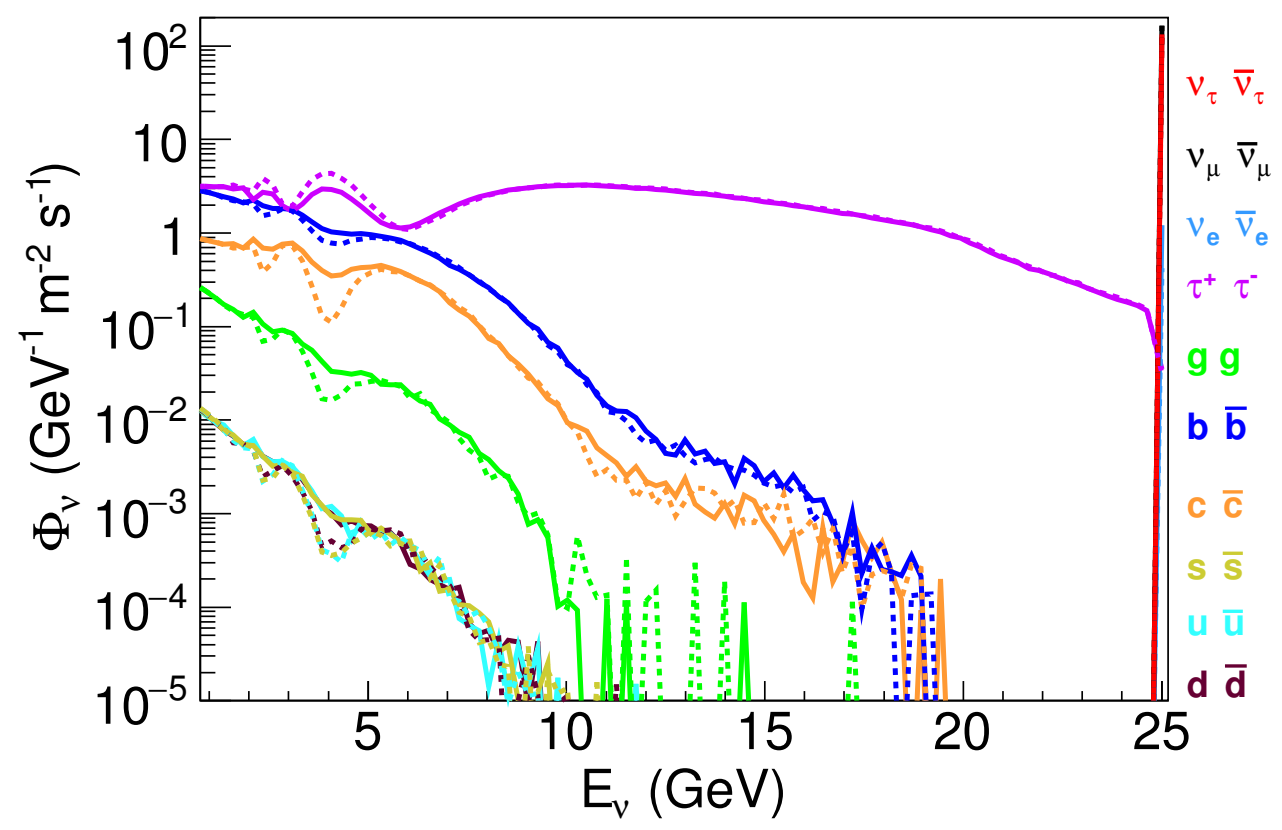

Figure 2. The $\nu$ and $\bar{\nu}$ fluxes at ICAL due to annihilation of $25 \mathrm{GeV}$ WIMP in the earth for various channels assuming $\sigma_{\mathrm{SI}}=10^{-38} \mathrm{~cm}^{2}$ and $\left\langle\sigma_{\mathrm{A}} v\right\rangle=3 \times 10^{-26} \mathrm{~cm}^{3} \mathrm{~s}^{-1}$.

neutrino-nucleon interactions. For the hadronisation, decay and production of neutrinos it uses PYTHIA [19]. For the results presented in this paper, we consider WIMP mass in the range $(10-100) \mathrm{GeV}$ and for $b^{-} b, \tau^{+} \tau^{-}$and $\nu_{\mu} \bar{\nu}_{\mu}$ annihilation channels. The propagation of neutrinos through earth matter involves neutrino oscillations which has been incorporated in a full three flavour neutrino framework with the oscillation parameters given in table 1. Throughout our analysis, we consider normal mass hierarchy.

WIMP can annihilate through various channels: $c \bar{c}, b \bar{b}, t \bar{t}, e^{+} e^{-}, \mu^{+} \mu^{-}, \tau^{+} \tau^{-}, W^{+} W^{-}$, $Z^{0} Z^{0}, g g, d \bar{d}, u \bar{u}$ and $s \bar{s}$. Among these channels, electrons are stable and the muons would interact and get absorbed inside the earth before they could produce high energy neutrinos. Therefore, electron and muon channels are not relevant to our analysis. The annihilation of WIMP into particles like protons, anti-deuterons, gamma rays will not produce neutrino fluxes, and hence such channels are also not considered in our work. The quark-antiquark annihilation channels like $u \bar{u}, d \bar{d}$ and $s \bar{s}$ will produce a weaker neutrino spectra and hence 
not competitive. For the WIMP mass range considered in this work, the channels $W^{+} W^{-}$, $Z^{0} Z^{0}, t \bar{t}$ are not kinematically relevant as they open up from masses $80.4 \mathrm{GeV}, 91.2 \mathrm{GeV}$ and $173 \mathrm{GeV}$, respectively. The annihilation to Higgs boson has also not been considered for the same reason.

Figure 2 shows the neutrino and antineutrino fluxes (in units of $\mathrm{GeV}^{-1} \mathrm{~m}^{-2} \Omega^{-1} \mathrm{~s}^{-1}$ ) at ICAL due to WIMP annihilations in the earth. For each of the annihilation channels, we assume $100 \%$ BR. We take $\rho_{\text {local }}=0.3 \mathrm{GeV} / \mathrm{cm}^{3}$ and $v_{\text {local }}=270 \mathrm{~km} \mathrm{sec}{ }^{-1}$ in our flux calculations. The fluxes shown in figure 2 are for $25 \mathrm{GeV}$ WIMP assuming a WIMP-nucleon scattering cross section $\sigma_{\mathrm{SI}}=10^{-38} \mathrm{~cm}^{2}$. We can see from the figure that the fluxes for both neutrinos as well as antineutrinos are nearly same, with antineutrino fluxes being slightly higher than the neutrino fluxes. This feature seems to hold for nearly all WIMP masses. The maximum kinematically possible energy of neutrinos produced from annihilation of WIMP is set by the WIMP mass i.e. for a WIMP mass of $25 \mathrm{GeV}$, the produced neutrinos will have energies in the range $(0-25) \mathrm{GeV}$. For the $\tau^{+} \tau^{-}$channel, the neutrino fluxes fall by about 1 order of magnitude in the above range. However, for the $b \bar{b}$ channel, the fluxes fall sharply by many orders of magnitude and well before $E_{\nu}=20 \mathrm{GeV}$ it becomes negligible. Therefore, stronger indirect detection bounds are expected with neutrino fluxes from $\tau^{+} \tau^{-}$channel in comparison to $b \bar{b}$ channel. We can see from the figure that the fluxes arising due to annihilation channels involving the other quark-antiquark pairs such as $c \bar{c}, d \bar{d}$, $u \bar{u}$ and $s \bar{s}$ are even weaker. Also shown are fluxes due to $g g$ channel and we can see that this channel is not competitive. Hence we do not consider those channels while discussing the expected sensitivity to indirect detection of dark matter in ICAL. Certain models, such as Kaluza-Klein (KK) [20], predicting WIMP annihilating directly into neutrino-antineutrino pairs can give rise to highly monoenergetic $\nu_{e} \bar{\nu}_{e}, \nu_{\mu} \bar{\nu}_{\mu}, \nu_{\tau} \bar{\nu}_{\tau}$ fluxes. Therefore, we expect a better sensitivity due to such channels. For the analysis carried out in this paper, we have considered the case of WIMP annihilating into $\nu_{\mu} \bar{\nu}_{\mu}$. The individual BR for a particular channel depends on a specific model considered. We, however, take a model-independent approach in this paper and quote the expected sensitivity limits for the $\tau^{+} \tau^{-}, b \bar{b}$ and $\nu_{\mu} \bar{\nu}_{\mu}$ channels with $100 \%$ BR each, as mentioned above. For a specific WIMP model, the flux with mixed BR for these channels will be between these extremes and hence the corresponding bounds. The figure 2 show fluxes for benchmark values of WIMP mass and cross-sections. The fluxes for other values of $\sigma_{\mathrm{SI}}$ can be obtained by simply scaling with the value of the cross-section. The above mentioned features of fluxes from various annihilation channels hold for all WIMP masses.

\section{Event generation at ICAL}

India-Based Neutrino Observatory (INO) is a proposed underground research facility to be built in Theni district of Tamil Nadu which is in the southern part of India. INO, among a few other experiments, will host be a $50 \mathrm{kt}$ Iron CALorimeter (ICAL) detector. ICAL will have 150 layers of glass Resistive Plate Chambers (RPCs) as an active medium. Each of these RPC layers will have iron plates between them which will act as an interaction medium where the muon neutrinos will interact with iron and produce muons. These muons will leave long tracks. Since the iron in the detector will be magnetised, $\mu^{+}$and 
$\mu^{-}$will bend in opposite directions giving ICAL a capability of charge identification [21]. ICAL, with its excellent angular resolution of muons, can be used to put limits on the WIMP annihilation from the earth, competitive with other indirect searches.

GENIE [22], suitably modified for our purpose, has been used for generating neutrino events with an ICAL geometry comprising $50 \mathrm{kt}$ iron mass and 150 layers of glass RPCs. We use fluxes calculated by Honda et al. [23] for Theni site for the simulation of atmospheric neutrino background. Signal calculation is done with the fluxes as prescribed in section 2 . Events are generated for a benchmark WIMP-nucleon cross-sections of $\sigma_{\mathrm{SI}}=10^{-44} \mathrm{~cm}^{2}$, assuming $100 \%$ BR for each of the annihilation channel, and then scaled appropriately for other WIMP-nucleon cross-sections.

We generate the signal and background events separately. Subsequently, we pass them through our reconstruction code whereby ICAL energy and angle resolutions, reconstruction and charge identification efficiencies are applied to get the final events at the detector. The muons, in our analysis, are binned in reconstructed energy and zenith angle bins. We perform detector simulations for ICAL geometry with Geant4 [24] and obtain the muon reconstruction efficiency, muon charge identification efficiency, muon zenith angle resolution and muon energy resolution values. We tabulate these resolutions and efficiencies in a two dimensional table implying that the muon energy and angle resolutions are a function of both muon energy as well as muon zenith angle and are described in detail in our previous work [8]. After incorporating the efficiencies and resolutions, the number of reconstructed $\mu^{-}$events in the $i j^{\text {th }}$ bin are:

$$
N_{i j}^{\prime \text { th }}=\mathcal{N} \sum_{k} \sum_{l} K_{i}^{k}\left(E_{T}^{k}\right) M_{j}^{l}\left(\cos \Theta_{T}^{l}\right)\left(\varepsilon_{k l} \mathcal{C}_{k l} n_{k l}\left(\mu^{-}\right)+\bar{\varepsilon}_{k l}\left(1-\overline{\mathcal{C}_{k l}}\right) n_{k l}\left(\mu^{+}\right)\right),
$$

where $\mathcal{N}$ is the normalisation that we require for a given exposure in ICAL. The summation in eq. (3.1) is over true muon energy and true muon zenith angle bins and are indicated by indices $k$ and $l$, respectively. $E_{T}$ and $\cos \Theta_{T}$ are the true (kinetic) energy and true zenith angle of the muon, respectively, whereas $E$ and $\cos \Theta$ are the corresponding reconstructed (kinetic) energy and zenith angle of reconstructed of $\mu^{-}$. Using the reweighting algorithm prescribed in [25], the raw $\mu^{-}$and $\mu^{+}$events from GENIE are folded with the three-generation oscillation probabilities and subsequently binned in terms of muon energy and zenith angles. The subsequent number of $\mu^{-}$and $\mu^{+}$events, in the respective $k^{\text {th }}$ true energy and $l^{\text {th }}$ true angle bin, are denoted by the quantities $n_{k l}\left(\mu^{-}\right)$and $n_{k l}\left(\mu^{+}\right)$. For the $k^{\text {th }}$ energy and the $l^{\text {th }}$ zenith angle bin, the quantities $\varepsilon_{k l}$ and $\bar{\varepsilon}_{k l}$ are the reconstruction efficiencies of $\mu^{-}$and $\mu^{+}$, respectively, and $\mathcal{C}_{k l}$ and $\overline{\mathcal{C}}_{k l}$ are the corresponding charge identification quantities. The reconstruction efficiencies as well as the charge identification efficiencies are the functions of the true muon energy $E_{T}$ and true muon zenith angle $\cos \Theta_{T}$. The Gaussian resolution functions $K_{i}^{k}$ and $M_{j}^{l}$ are used to apply muon energy and angle smearing respectively and are given as:

$$
\begin{aligned}
K_{i}^{k} & =\int_{E_{L_{i}}}^{E_{H_{i}}} d E \frac{1}{\sqrt{2 \pi} \sigma_{E}} \exp \left(-\frac{\left(E_{T}^{k}-E\right)^{2}}{2 \sigma_{E}^{2}}\right) \\
f M_{j}^{l}\left(\cos _{\Theta_{T}^{l}}\right) & =\int_{\cos \Theta_{L_{j}}}^{\cos \Theta_{H_{j}}} d \cos \Theta \frac{1}{\sqrt{2 \pi} \sigma_{\cos \Theta}} \exp \left(-\frac{\left(\cos \Theta_{T}^{k}-\cos \Theta\right)^{2}}{2 \sigma_{\cos \Theta}^{2}}\right) .
\end{aligned}
$$




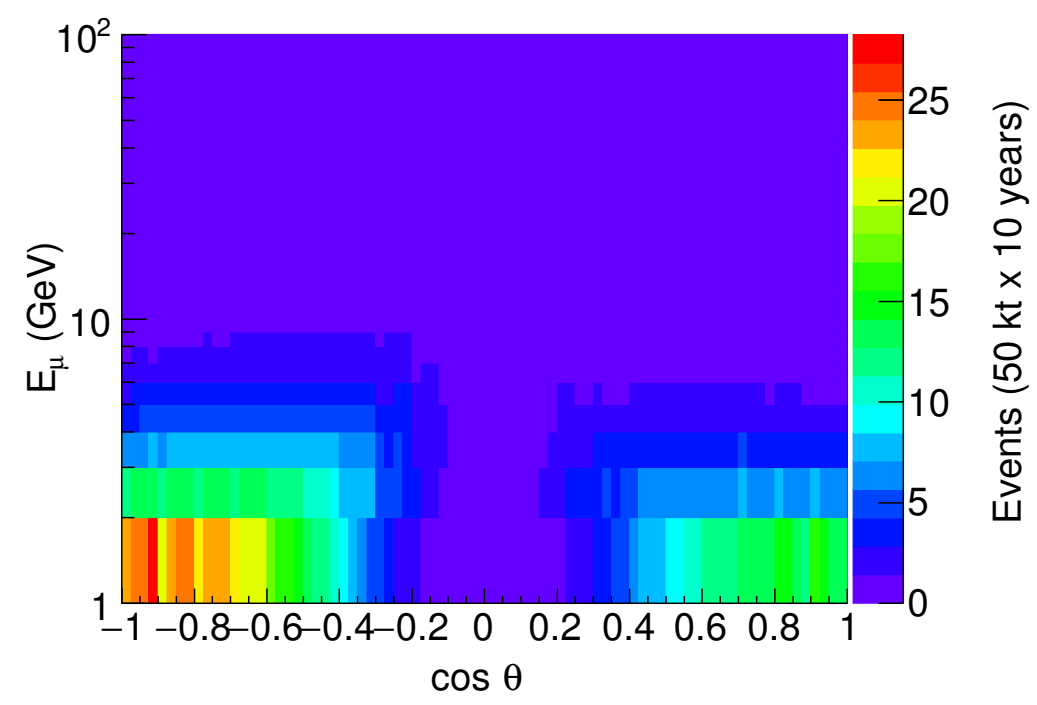

Figure 3. $\mu^{-}$event distribution at ICAL due to atmospheric neutrino background for $50 \times 10$ kt-years of ICAL exposure. ICAL has zero efficiency for horizontal tracks which is reflected in the bins around $\cos \theta=0$. Note that in ICAL convention $\cos \theta=1$ represents upward going muons.

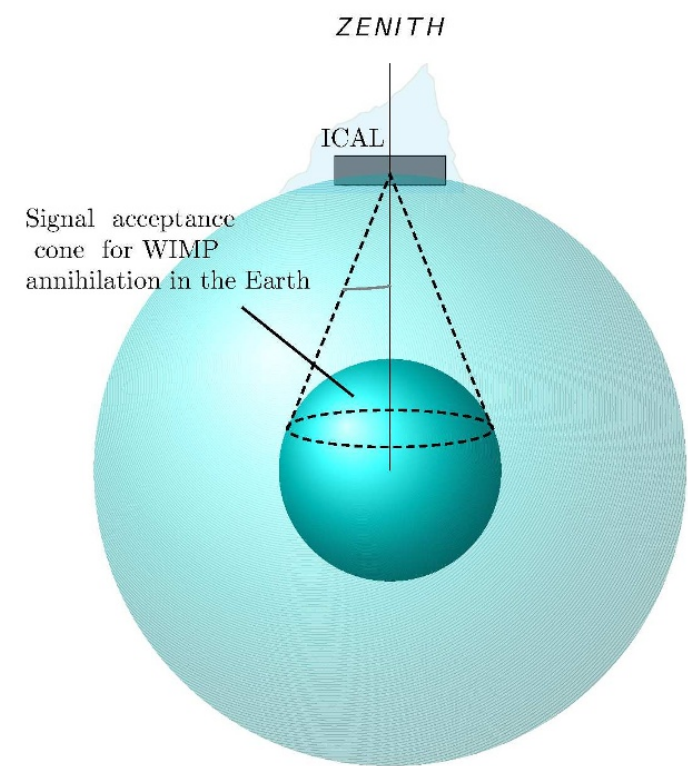

Figure 4. The cone regions where signal from the WIMP annihilations are expected for the earth.

The values of $\sigma_{E}$ and $\sigma_{\cos \Theta}$ are as given in appendix of [8]. Similar expressions can be written for the $\mu^{+}$events and $N_{i j}^{\prime \text { th }}\left(\mu^{+}\right)$. All analysis in this work is done for 10 years of ICAL running.

\section{Atmospheric neutrino background suppression}

The major source of background to the indirect searches of WIMP annihilation in the earth is due to the atmospheric neutrinos. However, unlike the neutrinos from WIMP 
annihilation which come from the direction of the earth core, the atmospheric neutrinos have a distribution over all zenith and azimuth angular bins and is comparatively well studied. We can exploit this feature and use it to suppress the atmospheric background considerably. Other source of neutrinos such a geothermal neutrinos coming from the core direction are in the $\mathrm{MeV}$ range and hence are not relevant here. The signal neutrinos, for the case of WIMP annihilation in the earth, will come from the direction of earth core. The signal search region for the WIMP annihilation in the earth is shown in figure 4 . These neutrinos on reaching ICAL will interact with the detector iron through charge current interaction and produce charged leptons, muons being the lepton of interest for ICAL. The scattered muon will make an angle $\left(\theta_{\nu \mu}\right)$ with their parent neutrino, where $\left(\theta_{\nu \mu}\right)$ is a function of parent $\nu$ energy and detector medium. Due to finite detector resolution there will be smearing effects. However, we choose to work with true muon direction rather than the reconstructed muon direction at the stage of background suppression. ICAL has an excellent muon angle resolution [8] for the considered energy range and hence this choice will not affect the final results significantly. We define $\theta_{90}$ to be the half angle of the cone that contains $90 \%$ of the signal muons, the axis of the cone being in the direction of the earth's centre. Harder channels like $\nu_{\mu} \bar{\nu}_{\mu}$ and $\tau^{+} \tau^{-}$with higher energy neutrinos will have a narrower $\theta_{90}$ in comparison to softer channels like $b \bar{b}$ with lower energy neutrinos which will have a broader $\theta_{90}$. Likewise, we expect that the neutrino spectra from annihilation of massive WIMP to have most of the associated muons in a narrower cones than neutrinos by lighter WIMP. Also, the heavier the WIMP, the closer it is to the centre of the earth and hence smaller cone opening ${ }^{1}$ Using WIMPSIM and GENIE, we estimate $\theta_{90}$ for each WIMP mass and for a given annihilation channel. Figure 5 shows the $\theta_{90}$ obtained for WIMP annihilation inside earth, as a function of WIMP mass $\left(m_{\chi}\right)$, and for different annihilation channels.

The atmospheric neutrino background is then suppressed as follows. For each WIMP mass and annihilation channel, we accept only those muons whose zenith angle are within $\theta_{90}$ with respect to the earth core. These atmospheric background events that fall within this cone represents an irreducible background for we can not distinguish them from the neutrinos due to WIMP annihilation in the core. After applying this suppression scheme, we fold the background events with detector resolution and efficiencies as described in the previous section 3 to obtain the final reconstructed and suppressed background events which are then used for $\chi^{2}$ analysis.

\footnotetext{
${ }^{1}$ The captured WIMP is expected to settle in core of the earth with an isothermal distribution at a temperature equal to the core temperature. In order to calculate WIMP annihilation profile for the earth, WIMPSIM uses the expression eq. (1) and (2) described in ref. [26]. At a distance $r$ from the center of the earth, the annihilation rate per unit volume is proportional to the square of the WIMP number density $n(r)$ and can be written as$$
n(r)=n(0) e^{-r^{2} / 2 r_{\chi}^{2}}
$$

where $r_{\chi}$ can be written as:

$$
r_{\chi}=\left[\frac{3 k T}{4 \pi G \rho m_{\chi}}\right]^{\frac{1}{2}} \simeq \frac{0.56 R_{\oplus}}{\sqrt{m_{\chi} / \mathrm{GeV}}} .
$$

In eq. $4.2, k$ and $G$ are the Boltzmann and Gravitational constants respectively, $R_{\oplus}$ is the radius of the earth, $\rho$ is the density of the earth centre and $T$ is the temperature at the centre of the earth.
} 

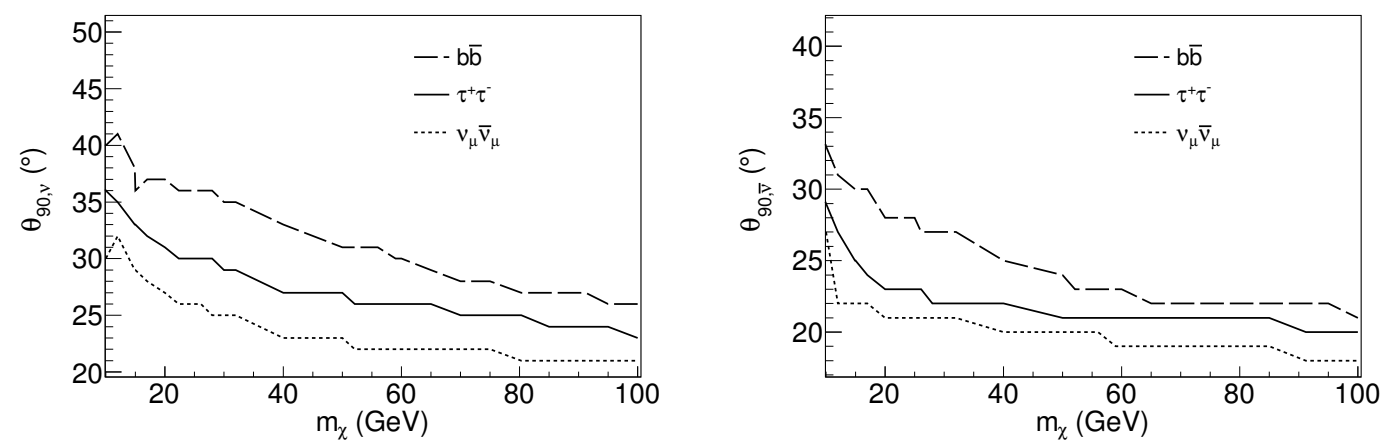

Figure 5. $90 \%$ cone cut values obtained for the earth, obtained using WIMPSIM and GENIE. A cone angle is estimated such that it contains $90 \%$ of the signal events. The LEFT panel shows the $\theta_{90}$ values for neutrinos and the RIGHT panel shows the corresponding values for anti-neutrinos, with different line types for the three WIMP annihilation channels: $\tau^{+} \tau^{-}$(solid), $b \bar{b}$ (dashed) and $\nu_{\mu} \bar{\nu}_{\mu}($ dotted $)$.

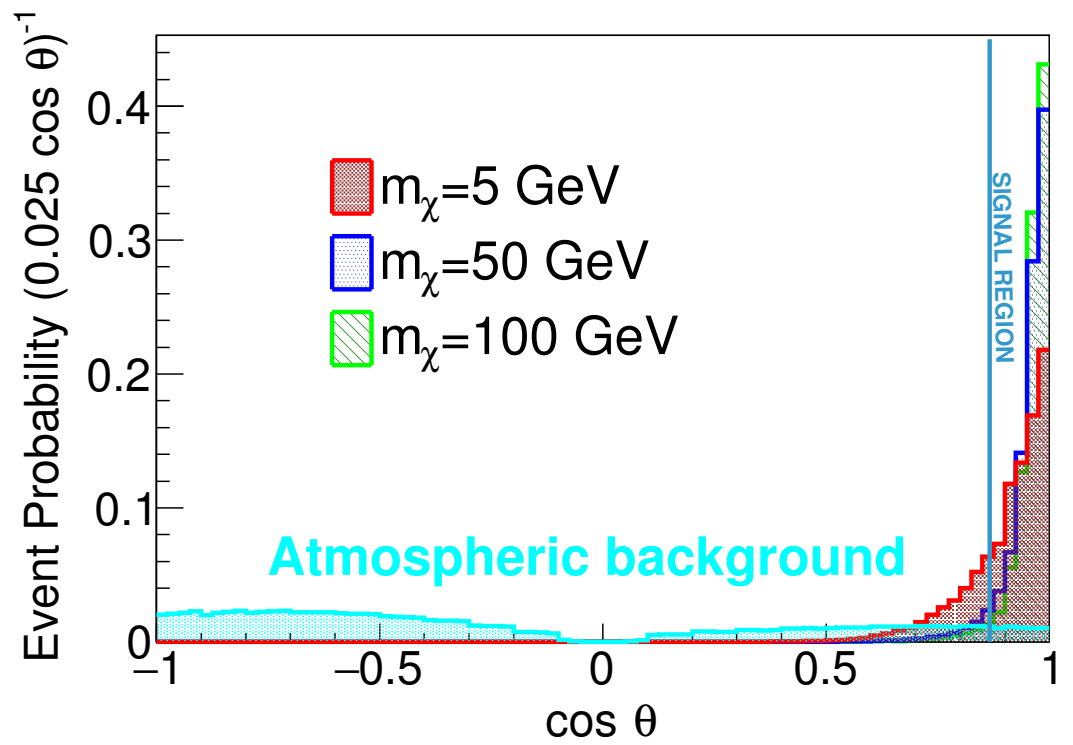

Figure 6. Angular probability distribution of reconstructed $\mu^{-}$at ICAL due to WIMP annihilations in the earth. A comparison for three WIMP masses has been shown and the region where signal is expected is marked. The probability distribution for the unsuppressed atmospheric background is also shown.

Figure 6 shows the angular probability distribution of $\mu^{-}$due to WIMP annihilations in the earth for the $\tau^{+} \tau^{-}$channel along with the distribution of the (unsuppressed) atmospheric background muon events at ICAL. A comparison for three WIMP masses 5, 50 and $100 \mathrm{GeV}$ has been shown. The above probability distribution, for each of the WIMP mass, has been obtained by normalising the reconstructed $\mu^{-}$events in each bin by total number of reconstructed $\mu^{-}$events for that WIMP mass. It can be noted that as WIMP mass increases, the angular probability distribution peaks towards the direction of the core. This is expected because of the reasons discussed above. The signal search is carried out 

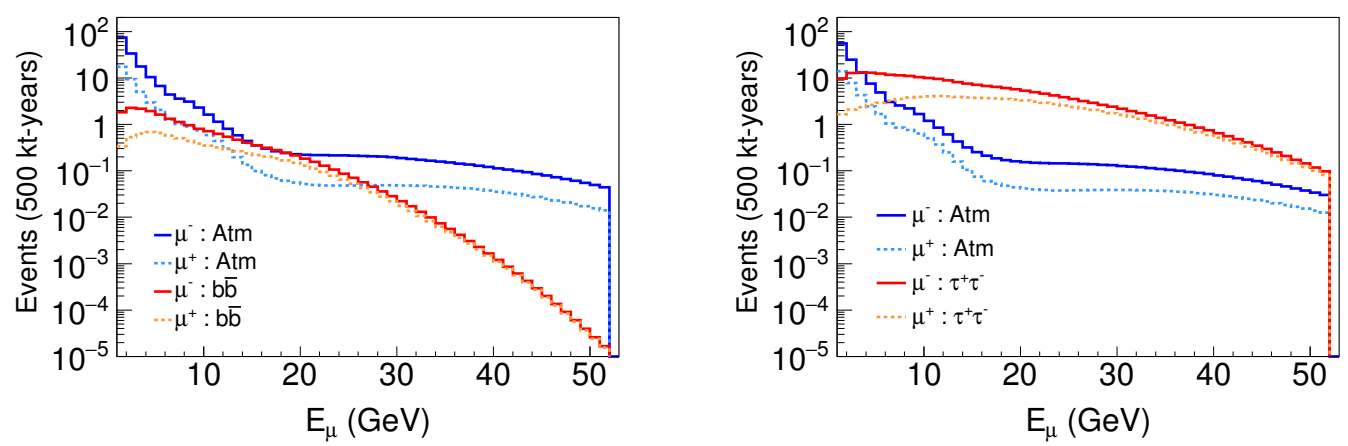

Figure 7. Muon event distribution at ICAL due to atmospheric neutrinos and signal neutrinos arising out of a WIMP annihilations in the earth for an assumed cross-section $\sigma_{\mathrm{SI}}=10^{-44} \mathrm{~cm}^{2}$. The left plot is for the WIMP annihilating into the channel $b \bar{b}$ while the right plot is for the annihilation channel $\tau^{+} \tau^{-}$. A $100 \%$ branching ratio has been assumed for each of the channels. Also shown are the corresponding events coming from the atmospheric background after applying the suppression scheme as described in 4. Atmospheric events have been simulated using Honda fluxes at Theni [27]. The softer channel $b \bar{b}$ has more background than the harder channel $\tau^{+} \tau^{-}$.

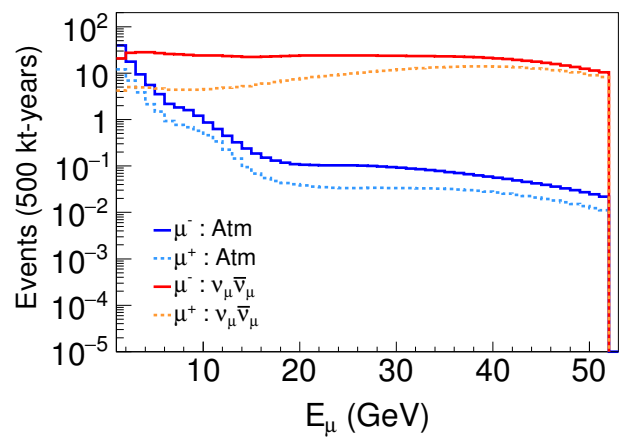

Figure 8. Muon event distribution at ICAL due to atmospheric neutrinos and signal neutrinos arising out of a WIMP annihilations in the earth for channel $\nu \bar{\nu}_{\mu}$ assuming $100 \%$ branching ratio and $\sigma_{\mathrm{SI}}=10^{-44} \mathrm{~cm}^{2}$. Also shown are the corresponding events coming from the atmospheric background after applying the suppression scheme as described in 4 .

in the region right of the vertical line represents $\theta_{90}$. We draw a line at $\sim 30^{\circ}$ from the earth centre $(\cos \theta=1)$ just for illustration. The actual values of $\theta_{90}$ for each WIMP mass and channel is taken from figure 5 while doing the analysis. Figure 7 shows the signal events due to a $52.14 \mathrm{GeV}$ WIMP annihilating through $\tau^{+} \tau^{-}$and $b \bar{b}$ channels and figure 8 due to $\nu_{\mu} \bar{\nu}_{\mu}$ annihilation channel. Also shown are corresponding suppressed atmospheric background events.

\section{The statistical analysis}

We estimate the $90 \%$ C.L. sensitivity limits on SI WIMP-nucleon cross-sections through a $\chi^{2}$ analysis. In our analysis, we generate prospective data at ICAL comprising atmospheric background events only. To this 'data', we fit our hypothesis in which we consider combined events predicated at ICAL due to WIMP annihilation in the earth and atmospheric neutrino 
background. This choice is consistent with 'no WIMP scenario' in the data and hence the limits calculated are the expected exclusion limits in the WIMP mass - WIMP SI crosssection plane from 10 years of running of ICAL.

We combine the $\mu^{+}$and $\mu^{-}$events while performing $\chi^{2}$ analysis. A $\chi^{2}$ function is defined as

$$
\chi^{2}=\chi^{2}\left(\mu^{-}\right)+\chi^{2}\left(\mu^{+}\right)
$$

where

$$
\begin{aligned}
\chi^{2}\left(\mu^{ \pm}\right)= & \min _{\xi_{k}^{ \pm}} \sum_{i=1}^{N_{i}} \sum_{j=1}^{N_{j}}\left[2\left(N_{i j}^{\mathrm{th}}\left(\mu^{ \pm}\right)-N_{i j}^{\mathrm{ex}}\left(\mu^{ \pm}\right)\right)+2 N_{i j}^{\mathrm{ex}}\left(\mu^{ \pm}\right) \ln \left(\frac{N_{i j}^{\mathrm{ex}}\left(\mu^{ \pm}\right)}{N_{i j}^{\mathrm{th}}\left(\mu^{ \pm}\right)}\right)\right] \\
& +\sum_{k=1}^{l} \xi_{k}^{ \pm 2} \\
N_{i j}^{\mathrm{th}}\left(\mu^{ \pm}\right)= & N_{i j}^{\prime \mathrm{th}}\left(\mu^{ \pm}\right)\left(1+\sum_{k=1}^{l} \pi_{i j}^{k} \xi_{k}^{ \pm}\right)+\mathcal{O}\left(\xi_{k}^{ \pm 2}\right)
\end{aligned}
$$

where $N_{i j}^{\prime \text { th }}\left(\mu^{ \pm}\right)$are the $\mu^{ \pm}$events that we 'predict' and $N_{i j}^{\text {ex }}\left(\mu^{ \pm}\right)$are the events 'observed' at ICAL. For $k^{\text {th }}$ systematic uncertainty we have associated $\pi_{i j}^{k}$ correction factors with $\xi_{k}^{ \pm}$ being the corresponding pull parameters. The quantity defined in eq. 5.3 is the predicted number of events including systematic uncertainties and is used in eq. 5.2 which defines the $\chi^{2}$ for Poisson distribution of errors. Similar to our previous analysis [28], we include 5 systematic errors as follows. We take $20 \%$ error on neutrino flux normalisation and $10 \%$ error on neutrino-nucleon cross-section. On the zenith angle distribution of atmospheric neutrino fluxes, we include a $5 \%$ uncorrelated error and $5 \%$ tilt error. Finally, we take a $5 \%$ overall error to account for detector systematics. Minimisation over the pull parameters gives the individual contributions from $\mu^{-}$and $\mu^{+}$data samples. Thereafter, we add them up and calculate the $\chi^{2}$ for a given set of WIMP mass and WIMP-nucleon cross-sections.

\section{Results}

We present the main results in this section. As mentioned in section 2, for the case of WIMP annihilation in the earth, the spin-independent WIMP nucleon cross sections $\sigma_{\mathrm{SI}}$ and annihilation rate $\Gamma_{\mathrm{A}}$ are related by eq. (2.2)-(2.6). The capture rate and the annihilation rate are not in equilibrium, and hence the annihilation rate depends on the $\sigma_{\mathrm{SI}}$ as well as on the annihilation cross section $\left\langle\sigma_{\mathrm{A}} v\right\rangle$. Figure 9 shows our expected sensitivity upper limits at $90 \%$ C.L. in the $\sigma_{\mathrm{SI}}-\left\langle\sigma_{\mathrm{A}} v\right\rangle$ plane for a WIMP mass of $50 \mathrm{GeV}$. As shown in figure 1, the capture rate for the WIMP masses closer to iron mass is greatly enhanced and hence we expect stronger bounds. It is evident that for a $50 \mathrm{GeV}$ WIMP, in comparison to other neutrino detectors, ICAL seems to put a stronger bound on $\sigma_{\mathrm{SI}}$ for a given $\left\langle\sigma_{\mathrm{A}} v\right\rangle$ for a given annihilation channel such as $\tau^{+} \tau^{-}$. For the obvious reasons, described in earlier sections, the harder channel $\nu_{\mu} \bar{\nu}_{\mu}$ gives a stronger limit in comparison to the softer channel $b \bar{b}$. The shaded region of the parameter space where equilibrium between the WIMP capture and annihilation in the earth has reached is calculated using eq. (2.6) and eq. (2.7). 


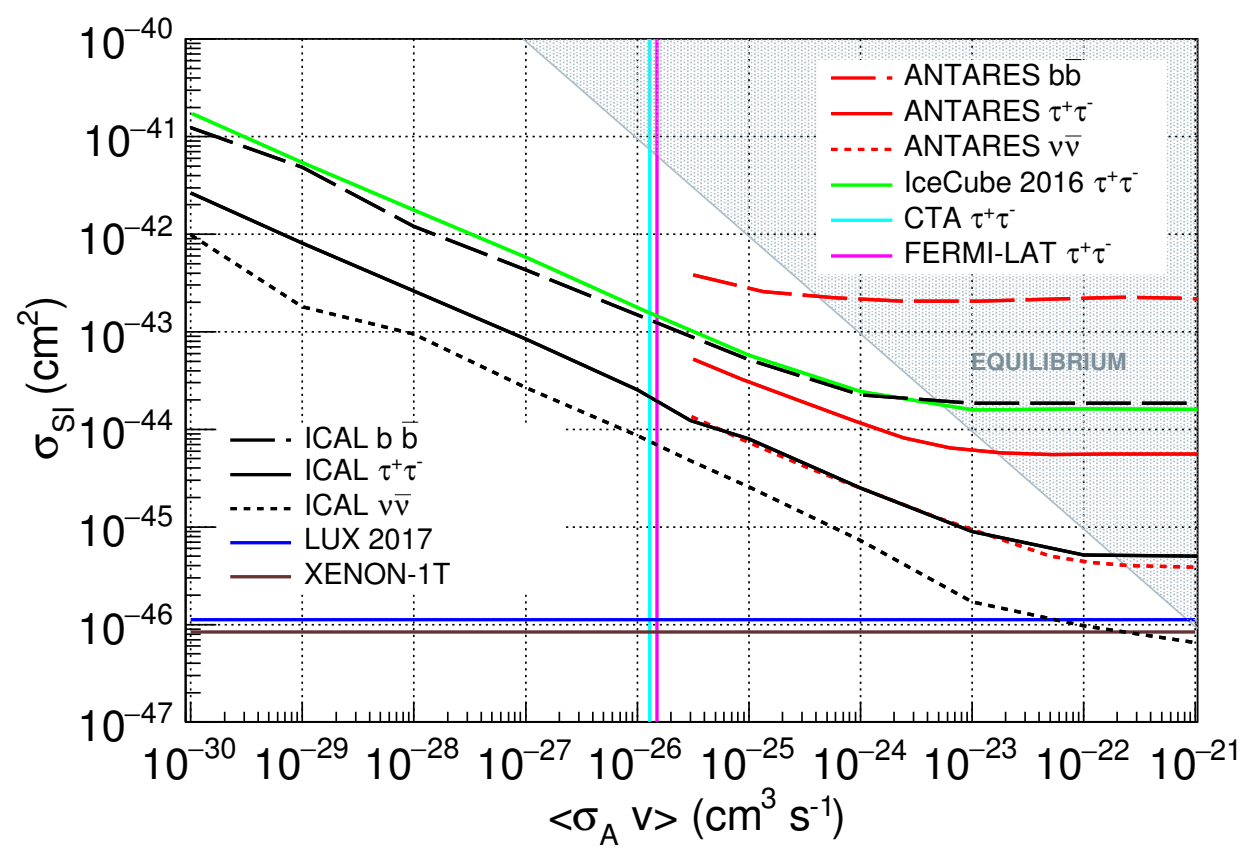

Figure 9. The expected $90 \%$ C.L. sensitivity limits on $\sigma_{\mathrm{SI}}$ for ICAL as a function of annihilation cross-section $\left\langle\sigma_{\mathrm{A}} v\right\rangle$ shown for a $50 \mathrm{GeV}$ WIMP annihilating through $b \bar{b}$ (black dashed), $\tau^{+}$and $\tau^{-}$ (black solid) and $\nu_{\mu} \bar{\nu}_{\mu}$ (black dotted) channels. The upper limits at $90 \%$ C.L. obtained by other neutrino experiments: IceCube [7] $\tau^{+}$and $\tau^{-}$(green), ANTARES [6] $\tau^{+}$and $\tau^{-}$(red solid), $b \bar{b}$ (red dashed), $\nu_{\mu} \bar{\nu}_{\mu}$ (red dotted) and direct detection experiments: LUX [29] (blue) and XENON1T [30] are shown for comparison. Exclusion limit at $95 \%$ C.L. for FERMI-LAT [31] (magenta) and estimated sensitivity at $95 \%$ C.L. of the upcoming CTA [32] (cyan) are also shown, where the region right to these limits are excluded. The region of the parameter space where equilibrium between the WIMP capture and annihilation in the earth has reached is marked as grey shaded. For ICAL, systematics have been included.

In figure 10 we present the expected $90 \%$ C.L. sensitivity limits on the WIMP-nucleon SI interaction cross-section as a function of WIMP mass for 500 kt-years of ICAL exposure and compare it with the exclusion limits obtained from various other direct and indirect detection experiments. The direct detection experiment XENON-1T [30] gives the most stringent bound till date. Bounds from indirect searches are, in general, weaker in comparison to direct detection experiments. However, among the neutrino detectors, ICAL seems to give the most stringent bound for the chosen WIMP mass range. For the WIMP masses close to iron mass, there is a resonant capture and hence enhance event rates resulting in a stronger bound.

For calculating sensitivity limits on annihilation rate and $\sigma_{\mathrm{SI}}$ in case of the earth, we assume an annihilation cross-section $\left\langle\sigma_{\mathrm{A}} v\right\rangle=3 \times 10^{-26} \mathrm{~cm}^{3} \mathrm{~s}^{-1}$. As discussed in section 2, we have a relation between the annihilation rate $\Gamma_{\mathrm{A}}$ and SI WIMP-nucleon cross-section $\sigma_{\mathrm{SI}}$. Using the $\chi^{2}$ analysis described in section 5 , we derive limits on the $\sigma_{\mathrm{SI}}$ as a function of WIMP mass $m_{\chi}$. Using eq. (2.2)-(2.6) from section 2, we transported these sensitivity limits from $\sigma_{\mathrm{SI}}-m_{\chi}$ plane to $\Gamma_{\mathrm{A}}-m_{\chi}$ plane. Figure 11 shows the expected sensitivity 


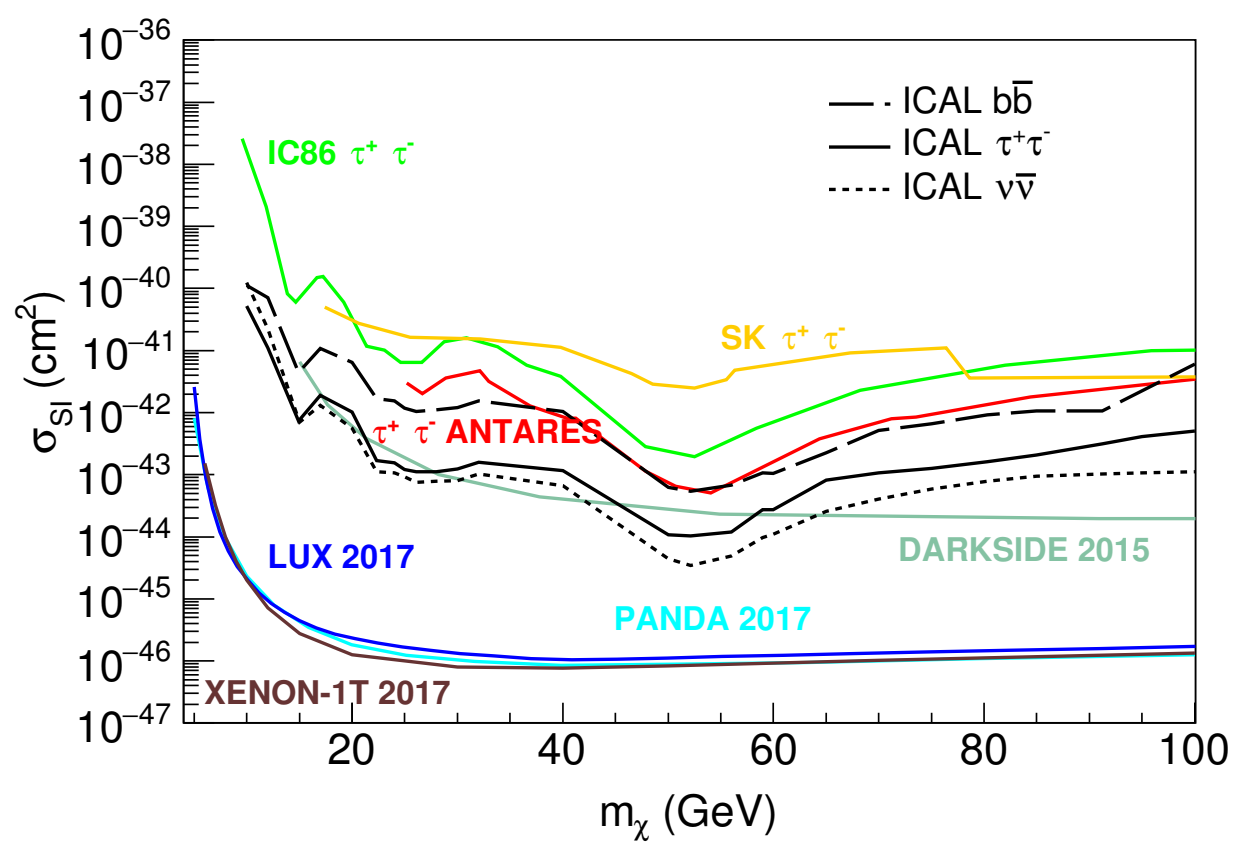

Figure 10. The expected $90 \%$ C.L. sensitivity limits on $\sigma_{\mathrm{SI}}$ as a function of WIMP mass, assuming a WIMP annihilation cross section $\left\langle\sigma_{\mathrm{A}} v\right\rangle=3 \times 10^{-26} \mathrm{~cm}^{3} \mathrm{~s}^{-1}$ are shown. The displayed sensitivity limits are for the local dark matter density $\rho=0.3 \mathrm{GeVcm}^{-3}$. Among the indirect detection experiments, involving neutrinos as a probe, ICAL provides the most stringent bound. The dip around $50 \mathrm{GeV}$ in the limits obtained for Earth WIMP annihilation is a prominent feature in all experiments and is due to resonant capture of WIMPs on Fe. ICAL 90\% C.L. sensitivity limits for $\tau^{+}$and $\tau^{-}$(black solid), $b \bar{b}$ (black dashed) and $\nu_{\mu} \bar{\nu}_{\mu}$ (black dotted) for WIMP annihilation in the earth are shown; 90\% C.L. upper limits from SK [33] for $\tau^{+}$and $\tau^{-}$(light orange), IceCube [7] $\tau^{+}$and $\tau^{-}$(green) and ANTARES [6] $\tau^{+}$and $\tau^{-}$(red). Also, shown are the limits obtained from DARKSIDE [34] (teal), LUX (blue) [29], XENON-1T [30] (brown) and PANDA [35] (cyan).

limits at $90 \%$ C.L. calculated on the WIMP annihilation rate for annihilation in the earth through channels $\nu_{\mu} \bar{\nu}_{\mu}, \tau^{+}$and $\tau^{-}$and $b \bar{b}$. Results from other experiments are also shown for comparison. ${ }^{2}$ Again, we can see that for chosen WIMP mass range, ICAL presents a stronger bound on the WIMP annihilation rate for a given channel. Again, $\nu_{\mu} \bar{\nu}_{\mu}$ and $\tau^{+} \tau^{-}$bounds are stronger than that of $b \bar{b}$ for the obvious reason that the channels with weaker neutrino fluxes are expected to give worse sensitivity limits for the corresponding WIMP masses. It should be noted that the results presented in this work assumes a particular annihilation cross section $\left\langle\sigma_{\mathrm{A}} v\right\rangle=3 \times 10^{-26} \mathrm{~cm}^{3} \mathrm{~s}^{-1}$. We expect the results would become proportionately weaker if the annihilation cross section is smaller (or stronger if it is larger). ${ }^{3}$

Finally, in figure 12 we present the effect of systematic uncertainties on the expected $90 \%$ C.L. sensitivity limits on the WIMP-nucleon SI interaction cross-section. The dashed lines are the limits calculated while taking only statistical uncertainties. As expected, the

\footnotetext{
${ }^{2}$ For SK latest preliminary results please see [36].

${ }^{3}$ Also see ref. [37] for more precise calculations of annihilation cross section.
} 


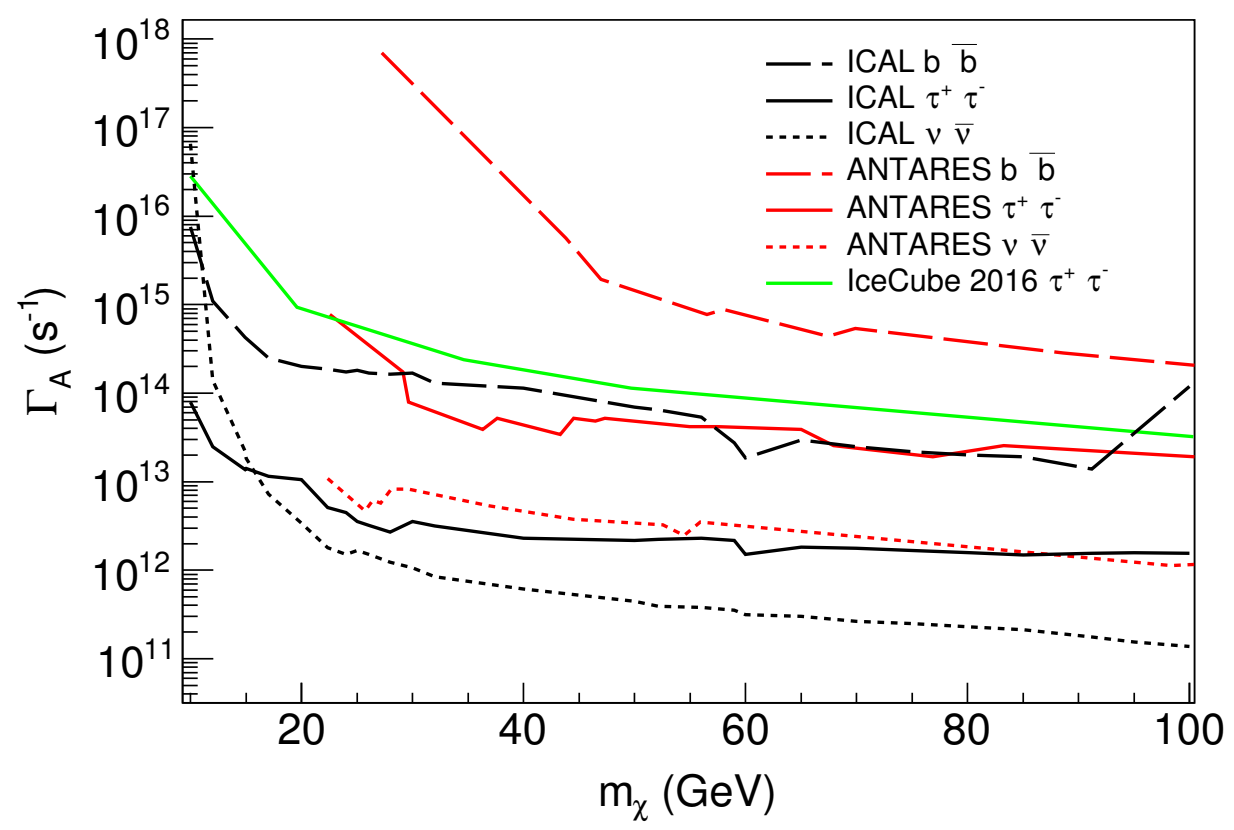

Figure 11. The expected $90 \%$ C.L. ICAL sensitivity limits on the annihilation rate $\left(\Gamma_{\mathrm{A}}\right)$ as function of WIMP mass $\left(m_{\chi}\right)$ due to WIMP annihilating into $\nu_{\mu} \bar{\nu}_{\mu}$ (black dotted), $\tau^{+} \tau^{-}$(black solid) and $b \bar{b}$ (black dashed), assuming $100 \%$ branching ratio for each channel, are shown. The limits are for 10 years of ICAL running. For comparison limits from various other experiments have been shown: ANATARES [6] $\tau^{+} \tau^{-}$(red solid), $b \bar{b}$ (red dashed), $\nu_{\mu} \bar{\nu}_{\mu}$ (red dotted), and IceCube [7] $\tau^{+}$and $\tau^{-}$for $m_{\chi}<50 \mathrm{GeV}$ and $W^{+} W^{-}$for $m_{\chi}>50 \mathrm{GeV}$ (green solid). We show these limits for a fixed $\left\langle\sigma_{\mathrm{A}} v\right\rangle=3 \times 10^{-26} \mathrm{~cm}^{3} \mathrm{~s}^{-1}$.

effect of systematic uncertainties is to worsen the limits as can be seen from the figure. However, the effect of systematics decreases for the harder channels and is almost negligible for the neutrino channel. This is expected since the harder the channel, the better is background suppression and hence lesser is the impact of systematic uncertainties.

\section{Summary}

The analysis presented in this work is a part of ongoing studies to probe the physics potential of the upcoming ICAL detector. Neutrinos arising out of WIMP annihilations in the earth could be used to probe dark matter signatures. We presented a study of prospects of detecting muon events at ICAL arising due to WIMP annihilation in the earth for $\tau^{+} \tau^{-}, b \bar{b}$ and $\nu_{\mu} \bar{\nu}_{\mu}$ annihilation channels. Employing an effective atmospheric background suppression scheme, the expected $90 \%$ C.L. sensitivity limits obtained for SI WIMP-nucleon cross-section for the case for the earth is better than any other neutrino detection experiment.

Such WIMP searches offer complementarity to direct detection approach. Firstly, a joint detection in ICAL and a direct-detection experiment would be useful in pinning down dark matter properties. A combined analysis of the data from ICAL and a direct-detection 


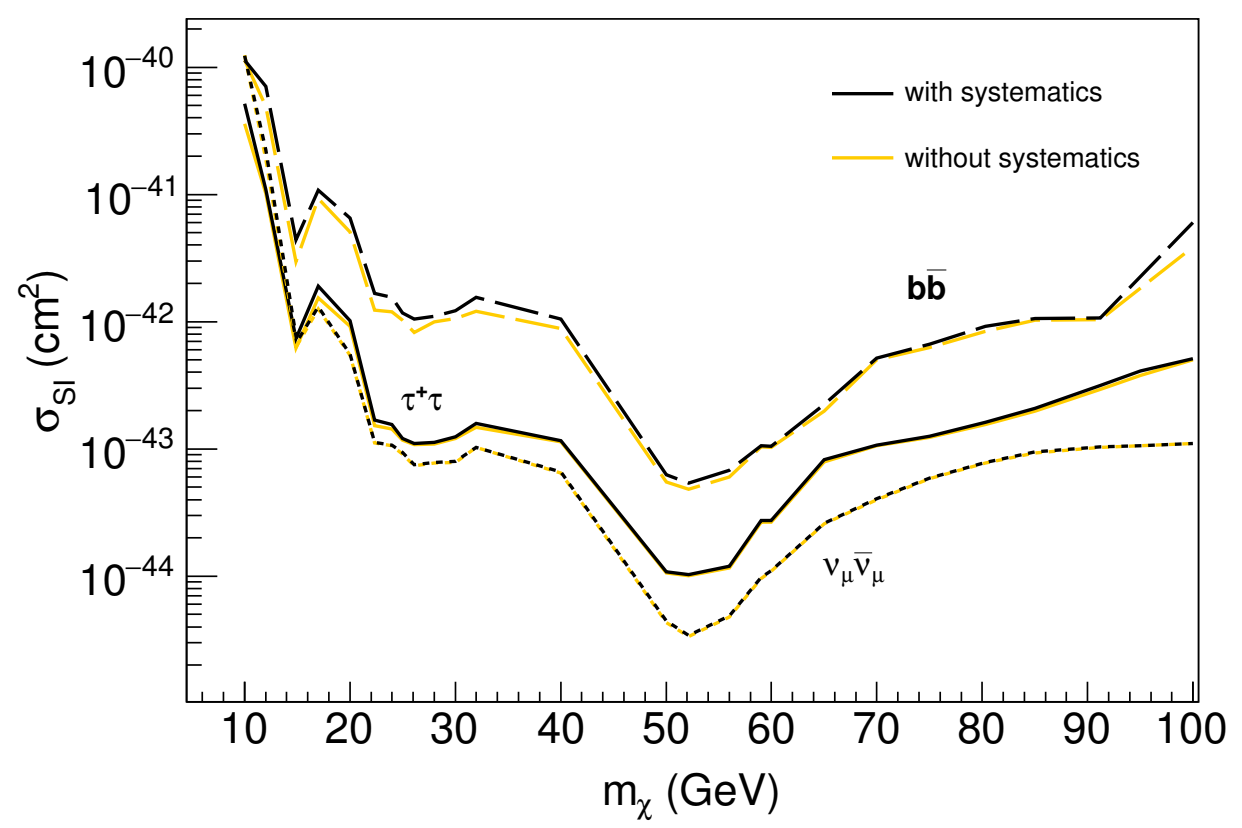

Figure 12. The expected 90\% C.L. sensitivity limits for WIMP annihilation in the earth for different masses and the three annihilation channels are shown. The black lines are the sensitivity limits calculated using detector systematics as described in section 5 . The corresponding orange lines are without systematics. The $90 \%$ C.L. expected sensitivity limits for $b \bar{b}$ are shown in black dashed (with systematics) and orange dashed lines (without systematics), for $\tau^{+}$and $\tau^{-}$in black solid (with systematics) and orange solid lines (without systematics) and for $\nu_{\mu} \bar{\nu}_{\mu}$ in black dotted (with systematics) and orange dotted lines (without systematics). The effect of systematics, as we expect, is to worsen the limits.

experiment can be useful in providing constraints on dark matter properties by cancelling out the astrophysical uncertainties such as those coming from lack of information on the correct local dark matter density and their local velocity distribution. Further, there could be dark matter models which might give signal in indirect detection experiments while remaining hidden from direct detection experiments and vice-versa. Finally, while both direct-detection experiments and ICAL are sensitive to WIMP nucleon scattering cross-section, for the latter case annihilation rate factors into the signal rate, resulting in sensitivity to WIMP annihilation cross-section. The extent of complementarity, or the extent to which combining ICAL data with other direct detection experiment data would help constrain dark matter cross section parameter space is under way and will be reported via a dedicated analyses in a future work.

\section{Acknowledgments}

We gratefully acknowledge INO collaboration for the support. We sincerely thank Amol Dighe for intensive discussions. DT wishes to thank Alan Robinson for the useful discussions. We acknowledge the HRI cluster computing facility (http://cluster.hri.res.in). The 
authors would like to thank the Department of Atomic Energy (DAE) Neutrino Project under the XII plan of Harish-Chandra Research Institute. This project has received funding from the European Union's Horizon 2020 research and innovation programme InvisiblesPlus RISE under the Marie Sklodowska-Curie grant agreement No 690575. This project has received funding from the European Union's Horizon 2020 research and innovation programme Elusives ITN under the Marie Sklodowska- Curie grant agreement No 674896. This project has received funding from FONDECYT 3170845 (Chile).

Open Access. This article is distributed under the terms of the Creative Commons Attribution License (CC-BY 4.0), which permits any use, distribution and reproduction in any medium, provided the original author(s) and source are credited.

\section{References}

[1] F. Zwicky, Die Rotverschiebung von extragalaktischen Nebeln, Helv. Phys. Acta 6 (1933) 110 [INSPIRE].

[2] G. Bertone, D. Hooper and J. Silk, Particle dark matter: evidence, candidates and constraints, Phys. Rept. 405 (2005) 279 [hep-ph/0404175] [INSPIRE].

[3] G. Steigman and M.S. Turner, Cosmological constraints on the properties of weakly interacting massive particles, Nucl. Phys. B 253 (1985) 375 [INSPIRE].

[4] ICECube collaboration, Search for annihilating dark matter in the Sun with 3 years of IceCube data, Eur. Phys. J. C 77 (2017) 146 [Erratum ibid. C 79 (2019) 214] [arXiv: 1612.05949] [INSPIRE].

[5] SUPER-KAMIOKANDE collaboration, Search for neutrinos from annihilation of captured low-mass dark matter particles in the Sun by Super-Kamiokande, Phys. Rev. Lett. 114 (2015) 141301 [arXiv: 1503.04858] [INSPIRE].

[6] ANTARES collaboration, Search for dark matter annihilation in the Earth using the ANTARES neutrino telescope, Phys. Dark Univ. 16 (2017) 41 [arXiv:1612.06792] [INSPIRE].

[7] ICECuBE collaboration, First search for dark matter annihilations in the Earth with the IceCube detector, Eur. Phys. J. C 77 (2017) 82 [arXiv: 1609.01492] [INSPIRE].

[8] S. Choubey, A. Ghosh and D. Tiwari, Prospects of indirect searches for dark matter at INO, JCAP 05 (2018) 006 [arXiv: 1711.02546] [INSPIRE].

[9] G. Jungman, M. Kamionkowski and K. Griest, Supersymmetric dark matter, Phys. Rept. 267 (1996) 195 [hep-ph/9506380] [INSPIRE].

[10] D.N. Spergel and W.H. Press, Effect of hypothetical, weakly interacting, massive particles on energy transport in the solar interior, Astrophys. J. 294 (1985) 663 [INSPIRE].

[11] A. Gould, WIMP distribution in and evaporation from the Sun, Astrophys. J. 321 (1987) 560 [INSPIRE].

[12] L.M. Krauss, M. Srednicki and F. Wilczek, Solar system constraints and signatures for dark matter candidates, Phys. Rev. D 33 (1986) 2079 [INSPIRE].

[13] K. Griest and D. Seckel, Cosmic asymmetry, neutrinos and the Sun, Nucl. Phys. B 283 (1987) 681 [Erratum ibid. B 296 (1988) 1034] [INSPIRE].

[14] A. Gould, Resonant enhancements in WIMP capture by the Earth, Astrophys. J. 321 (1987) 571 [INSPIRE]. 
[15] A. Gould, Cosmological density of WIMPs from solar and terrestrial annihilations, Astrophys. J. 388 (1992) 338 [INSPIRE].

[16] M. Blennow, J. Edsjo and T. Ohlsson, Neutrinos from WIMP annihilations using a full three-flavor Monte Carlo, JCAP 01 (2008) 021 [arXiv:0709.3898] [INSPIRE].

[17] J. Edsjo, WimpSim Neutrino Monte Carlo, http://www.fysik.su.se/edsjo/wimpsim.

[18] J. Edsjo, NuSigma neutrino interaction Monte Carlo, http://www.fysik.su.se/edsjo/wimpsim.

[19] T. Sjöstrand, S. Mrenna and P.Z. Skands, PYTHIA 6.4 physics and manual, JHEP 05 (2006) 026 [hep-ph/0603175] [INSPIRE].

[20] M. Blennow, H. Melbeus and T. Ohlsson, Neutrinos from Kaluza-Klein dark matter in the Sun, JCAP 01 (2010) 018 [arXiv:0910.1588] [INSPIRE].

[21] ICAL collaboration, Physics potential of the ICAL detector at the India-based Neutrino Observatory (INO), Pramana 88 (2017) 79 [arXiv: 1505.07380] [INSPIRE].

[22] C. Andreopoulos et al., The GENIE neutrino Monte Carlo generator, Nucl. Instrum. Meth. A 614 (2010) 87 [arXiv:0905.2517] [InSPIRE].

[23] M. Honda, T. Kajita, K. Kasahara and S. Midorikawa, Improvement of low energy atmospheric neutrino flux calculation using the jam nuclear interaction model, Phys. Rev. D 83 (2011) 123001.

[24] GEANT4 collaboration, GEANT4: a Simulation toolkit, Nucl. Instrum. Meth. A 506 (2003) 250 [INSPIRE].

[25] A. Ghosh, T. Thakore and S. Choubey, Determining the neutrino mass hierarchy with INO, T2K, NOvA and reactor experiments, JHEP 04 (2013) 009 [arXiv: 1212.1305] [INSPIRE].

[26] J. Edsjo and P. Gondolo, WIMP mass determination with neutrino telescopes, Phys. Lett. B 357 (1995) 595 [hep-ph/9504283] [INSPIRE].

[27] M. Honda et al., Atmospheric neutrino flux calculation using the NRLMSISE-00 atmospheric model, Phys. Rev. D 92 (2015) 023004 [arXiv:1502.03916] [INSPIRE].

[28] S. Choubey, A. Ghosh, T. Ohlsson and D. Tiwari, Neutrino physics with non-standard interactions at INO, JHEP 12 (2015) 126 [arXiv:1507.02211] [INSPIRE].

[29] LUX collaboration, Results from a search for dark matter in the complete LUX exposure, Phys. Rev. Lett. 118 (2017) 021303 [arXiv:1608.07648] [inSPIRE].

[30] XENON collaboration, First dark matter search results from the XENON1T experiment, Phys. Rev. Lett. 119 (2017) 181301 [arXiv:1705. 06655] [INSPIRE].

[31] Fermi-LAT collaboration, Searching for dark matter annihilation from Milky Way dwarf spheroidal galaxies with six years of Fermi Large Area Telescope data, Phys. Rev. Lett. 115 (2015) 231301 [arXiv:1503.02641] [INSPIRE].

[32] H. Silverwood, C. Weniger, P. Scott and G. Bertone, A realistic assessment of the CTA sensitivity to dark matter annihilation, JCAP 03 (2015) 055 [arXiv:1408.4131] [INSPIRE].

[33] Super-Kamiokande collaboration, Search for dark matter WIMPs using upward through-going muons in Super-Kamiokande, Phys. Rev. D 70 (2004) 083523 [Erratum ibid. D 70 (2004) 109901] [hep-ex/0404025] [INSPIRE].

[34] DARKSIDE collaboration, First results from the DarkSide-50 dark matter experiment at Laboratori Nazionali del Gran Sasso, Phys. Lett. B 743 (2015) 456 [arXiv:1410.0653] [INSPIRE]. 
[35] PandaX-II collaboration, Dark matter results from 54-ton-day exposure of PandaX-II experiment, Phys. Rev. Lett. 119 (2017) 181302 [arXiv:1708.06917] [InSPIRE].

[36] Super-Kamiokande collaboration, Dark matter searches with the Super-Kamiokande detector, J. Phys. Conf. Ser. 888 (2017) 012210.

[37] G. Steigman, B. Dasgupta and J.F. Beacom, Precise relic WIMP abundance and its impact on searches for dark matter annihilation, Phys. Rev. D 86 (2012) 023506 [arXiv:1204.3622] [INSPIRE]. 